\title{
Correction to: How Does Administrative Data \\ Compare with Census Data for the Measurement of Internal Migration? The Case of Northern Ireland, 2001-2011
}

\author{
Brian Foley ${ }^{1} \cdot$ Tony Champion $^{2} \cdot \operatorname{lan}$ Shuttleworth $^{3}$ (B) \\ Published online: 11 August 2021 \\ (C) Springer Nature B.V. 2021
}

\section{Correction to: Applied Spatial Analysis and Policy https://doi.org/10.1007/s12061-020-09369-w}

The original version of this article unfortunately contains missing data in Appendix Table 2.

The corrected Appendix Table 2 is shown below.

The online version of the original article can be found at https://doi.org/10.1007/s12061-020-09369-w

\author{
Ian Shuttleworth \\ i.shuttleworth@qub.ac.uk \\ Brian Foley \\ Brian.foley@nisra.gov.uk \\ Tony Champion \\ Tony.champion@newcastle.ac.uk
}

1 Northern Ireland Statistics and Research Agency, Belfast, Northern Ireland

2 Newcastle University, Newcastle, UK

3 Queen's University Belfast, Belfast, Northern Ireland 


\section{Appendix}

Table 2 Odds Ratios, Coefficients and Standards Errors and Confidence Limits Underlying Fig. 6. Source: NILS

\begin{tabular}{|c|c|c|c|c|c|c|c|c|c|c|}
\hline & \multicolumn{5}{|l|}{$\mathrm{HCR}$} & \multicolumn{5}{|c|}{ Census } \\
\hline & Coeff & \multirow[t]{2}{*}{$\begin{array}{l}\text { Odds } \\
\text { Ratio }\end{array}$} & \multirow[t]{2}{*}{$\begin{array}{l}\text { Std. } \\
\text { Err. }\end{array}$} & \multicolumn{2}{|c|}{$\begin{array}{l}\text { [95\% Conf. } \\
\text { Interval] }\end{array}$} & \multirow[t]{2}{*}{ Coeff } & \multirow[t]{2}{*}{$\begin{array}{l}\text { Odds } \\
\text { Ratio }\end{array}$} & \multirow[t]{2}{*}{$\begin{array}{l}\text { Std. } \\
\text { Err. }\end{array}$} & \multicolumn{2}{|c|}{$\begin{array}{l}\text { [95\% Conf. } \\
\text { Interval] }\end{array}$} \\
\hline \multicolumn{6}{|l|}{ Age } & & & & & \\
\hline Ref: 55-64 & & 1.000 & & & & & 1.000 & & & \\
\hline $16-24$ & 1.391 & 4.019 & 0.091 & 3.844 & 4.202 & 1.822 & 6.182 & 0.146 & 5.903 & 6.475 \\
\hline $25-34$ & 1.856 & 6.399 & 0.116 & 6.177 & 6.630 & 2.602 & 13.487 & 0.259 & 12.989 & 14.004 \\
\hline $35-44$ & 1.529 & 4.611 & 0.077 & 4.464 & 4.764 & 1.770 & 5.868 & 0.100 & 5.676 & 6.067 \\
\hline $45-54$ & 0.530 & 1.699 & 0.026 & 1.649 & 1.752 & 0.592 & 1.808 & 0.028 & 1.753 & 1.864 \\
\hline $65-74$ & -0.218 & 0.804 & 0.015 & 0.776 & 0.833 & -0.156 & 0.856 & 0.016 & 0.825 & 0.887 \\
\hline \multicolumn{11}{|l|}{ Gender } \\
\hline Ref: Male & & 1.000 & & & & & 1.000 & & & \\
\hline Female & 0.095 & 1.100 & 0.010 & 1.081 & 1.118 & -0.015 & 0.985 & 0.009 & 0.968 & 1.003 \\
\hline \multicolumn{11}{|l|}{ Marital status } \\
\hline Ref: Married & & 1.000 & & & & & 1.000 & & & \\
\hline Single & -0.824 & 0.439 & 0.007 & 0.426 & 0.452 & -0.986 & 0.373 & 0.006 & 0.362 & 0.385 \\
\hline $\begin{array}{l}\text { Separated, } \\
\text { Divorced,Widowed }\end{array}$ & 0.246 & 1.279 & 0.019 & 1.242 & 1.317 & 0.204 & 1.226 & 0.019 & 1.189 & 1.264 \\
\hline \multicolumn{11}{|l|}{$\mathrm{CoB}$} \\
\hline Ref: UK/RoI & & 1.000 & & & & & 1.000 & & & \\
\hline Non-UK/ROI & 0.248 & 1.282 & 0.049 & 1.189 & 1.382 & 0.210 & 1.234 & 0.048 & 1.142 & 1.333 \\
\hline \multicolumn{11}{|l|}{ NS-Sec } \\
\hline Ref: Intermediate & & 1.000 & & & & & 1.000 & & & \\
\hline Prof & 0.134 & 1.144 & 0.013 & 1.118 & 1.170 & 0.157 & 1.170 & 0.014 & 1.143 & 1.197 \\
\hline Routine & -0.117 & 0.889 & 0.010 & 0.870 & 0.910 & -0.215 & 0.807 & 0.010 & 0.788 & 0.826 \\
\hline Not working & -0.056 & 0.945 & 0.018 & 0.911 & 0.981 & -0.238 & 0.788 & 0.016 & 0.758 & 0.820 \\
\hline Student & -0.181 & 0.835 & 0.017 & 0.802 & 0.869 & -0.273 & 0.761 & 0.016 & 0.731 & 0.794 \\
\hline \multicolumn{11}{|l|}{ Housing tenure } \\
\hline Ref: Owner occ & & 1.000 & & & & & 1.000 & & & \\
\hline Soc rent & 0.921 & 2.513 & 0.037 & 2.442 & 2.585 & 1.091 & 2.978 & 0.044 & 2.893 & 3.067 \\
\hline Priv rent & 1.499 & 4.477 & 0.071 & 4.340 & 4.619 & 2.617 & 13.693 & 0.283 & 13.149 & 14.259 \\
\hline CE Other & 0.651 & 1.918 & 0.069 & 1.787 & 2.058 & 1.022 & 2.778 & 0.100 & 2.590 & 2.981 \\
\hline \multicolumn{11}{|l|}{ Household composition } \\
\hline Ref: Family, dep children & & 1.000 & & & & & 1.000 & & & \\
\hline Other & -0.193 & 0.824 & 0.010 & 0.805 & 0.844 & -0.011 & 0.989 & 0.012 & 0.965 & 1.013 \\
\hline Cohab & 0.559 & 1.748 & 0.039 & 1.674 & 1.826 & 1.381 & 3.979 & 0.100 & 3.789 & 4.179 \\
\hline Lone par & -0.033 & 0.967 & 0.016 & 0.936 & 1.000 & -0.024 & 0.976 & 0.017 & 0.944 & 1.010 \\
\hline Stud HH & -0.891 & 0.410 & 0.032 & 0.352 & 0.477 & 3.834 & 46.253 & 19.261 & 20.450 & 104.615 \\
\hline $\mathrm{CE} /$ vacant & 0.920 & 2.508 & 0.180 & 2.180 & 2.886 & 3.308 & 27.344 & 3.037 & 21.995 & 33.993 \\
\hline
\end{tabular}

Area type 
Table 2 (continued)

\begin{tabular}{|c|c|c|c|c|c|c|c|c|c|c|}
\hline & \multicolumn{5}{|l|}{$\mathrm{HCR}$} & \multicolumn{5}{|l|}{ Census } \\
\hline & Coeff & $\begin{array}{l}\text { Odds } \\
\text { Ratio }\end{array}$ & \multirow[t]{2}{*}{$\begin{array}{l}\text { Std. } \\
\text { Err. }\end{array}$} & \multicolumn{2}{|c|}{$\begin{array}{l}\text { [95\% Conf. } \\
\text { Interval] }\end{array}$} & Coeff & $\begin{array}{l}\text { Odds } \\
\text { Ratio }\end{array}$ & \multirow[t]{2}{*}{$\begin{array}{l}\text { Std. } \\
\text { Err. }\end{array}$} & \multicolumn{2}{|c|}{$\begin{array}{l}\text { [95\% Conf. } \\
\text { Interval] }\end{array}$} \\
\hline Ref: Rural & & 1.000 & & & & & 1.000 & & & \\
\hline Urban & 0.079 & 1.083 & 0.010 & 1.062 & 1.103 & -0.008 & 0.992 & 0.010 & 0.973 & 1.012 \\
\hline \multicolumn{11}{|l|}{ Social deprivation } \\
\hline Ref: 1 - least deprived & & 1.000 & & & & & 1.000 & & & \\
\hline 2 & -0.038 & 0.962 & 0.012 & 0.939 & 0.986 & -0.034 & 0.967 & 0.012 & 0.943 & 0.991 \\
\hline 3 & -0.161 & 0.851 & 0.010 & 0.831 & 0.872 & -0.226 & 0.798 & 0.010 & 0.778 & 0.818 \\
\hline Most deprived & -0.227 & 0.797 & 0.010 & 0.777 & 0.817 & -0.276 & 0.759 & 0.010 & 0.739 & 0.779 \\
\hline Constant & -1.330 & 0.264 & 0.005 & 0.255 & 0.275 & -1.418 & 0.242 & 0.005 & 0.233 & 0.252 \\
\hline
\end{tabular}

Publisher's Note Springer Nature remains neutral with regard to jurisdictional claims in published maps and institutional affiliations. 